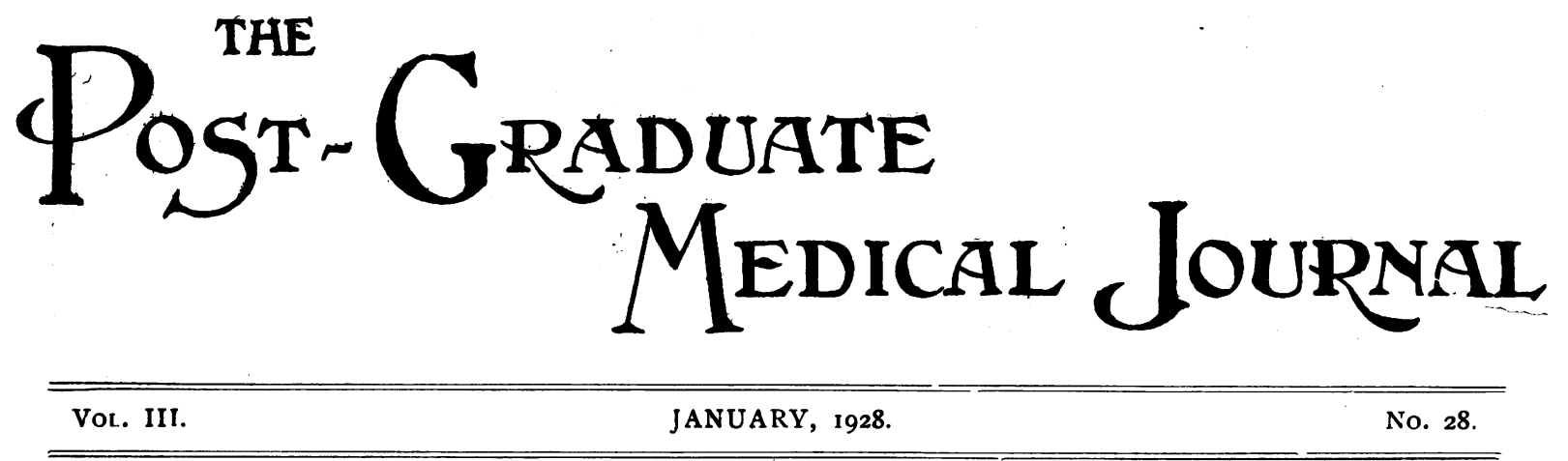

\title{
CONTENTS
}

SURGical Hints.

By Herbert J. Paterson, C.B.E., M.C., M.D., F.R.C.S. $\quad \ldots \quad \ldots \quad \ldots \quad \ldots 5$

The USES and Limitations of Light Treathent.

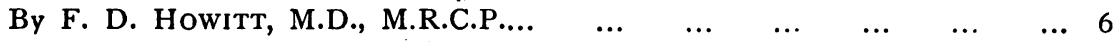

The General Principles of Nutrition.

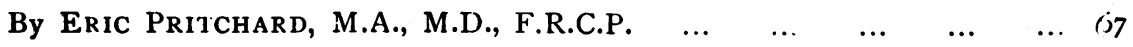

$\begin{array}{llllllllllll}\text { EDITORIAL NOTES } & \ldots & \ldots & \ldots & \ldots & \ldots & \ldots & \ldots & \ldots & \ldots & \ldots & 70\end{array}$

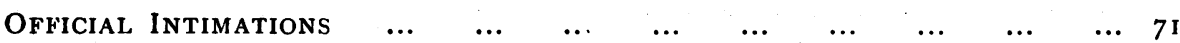

Fellowship of Medicine and Post-Graduate Medical Association.-

$\begin{array}{llllllllll}\text { SPECIAL Courses } \ldots & \ldots & \ldots & \ldots & \ldots & \ldots & \ldots & \ldots & \ldots & \text { iii, }\end{array}$

\section{SURGICAL HINTS.}

A LECTURE DELIVERED

\section{By HERBERT J. PATERSON,}

C.B.E., M.C., M.D., F.R.C.S.

(Under the Auspices of The Fellowship of Medicine),

AT THE ROOMS OF THE MEDICAL SOCIETY OF LONDON; ON OCTOBER 24, 1927.

LAdies and Gentlemen,-I do not propose to give you a formal lecture, but a series of "tips" in suigery, which ,I hope will be of use to you. I know most of you have got to the stage at which you realize that you can always learn something more. I dare say many of you remember when you were first qualified you thought you knew everything, and that when one's name was included in the list of house surgeons at the hospital, one was then really "It." When, however, a case came along which you could not quite fathom, you began to feel a little less confident in yourself. When, in addition, a Sister "put you wise" over a case which had been puzzling you, you realized that there was yet much for you to learn. I think it is most important for postgraduates when they go into practice to keep up to date and keep in touch with hospital work, because, after all, undergraduate studies are simply the foundation on which the superstructure of knowledge and experience has to be built during the remaining years of our life. We are never too old to learn; the longer we are in practice the longer we go on learning something. It is only the knowledge and experience which we gain after we are qualified 
that enable us to diagnose and treat disease. I think it was Sir William Osler who once said : "A doctor without a post-graduate course is stale in five years, in a rut in ten years, out of which only a prolonged course will remove him, and in twenty years, in a well out of which nothing will drag him."

\section{LABORATORY DIAGNOSIS.}

My first "tip" has reference to the socalled modern scientific surgery, the surgery which we are all supposed to practise to day. It is this: Do not try to have your diagnosis made for you in the laboratory or the X-ray department. I know that is the modern way of doing things, and I am afraid that if we go on in the way we are doing, clinical diagnosis will soon become a linst art. It is quite common, in hospital work, to ask one's house surgeon what sort of displacement there is in a fracture, and for him to tell one, quite openly and without blushinis, that he does not know, but he has sent the case to the X-ray department, to have a skiagram taken. But that is not surgery. The surgeon ought to be able to tell quite as much as the radiographer can, perhaps more. Therefore I say: First, make your own diagnosis, then, if necessary, call in the aid of the laboratory to confirm it; and unless the laboratory diagnosis agrees with your own, do not pay any attention to it. Do not be a slave to laboratory investigations; they are useful servants, but very bad masters. Americans put it very pithily when they say, "Don't let the pathologist wag your tail." Every surgeon ought to wag his own tail. We are too ready to accept as gospel truth the diagnoses which are made for us in the laboratory and the $\mathrm{X}$-ray room. You cannot make a diagnosis in a test-tube, because surgery is an art, rather than a science.

Surgery is an art, and you cannot turn it into a science, it is not exact enough. If it were not an art, one doctor would be as good as another; but some are better than others, because they have a greater mastery of the art. To know when to operate is more important than to know how to operate. It is said that Demosthenes was asked what were the chief attributes necessary for an orator, and his reply was, "First, action; secondly, action ; thirdly, action"; and I think we might well adopt his method of emphasis, and say that the three most important attributes of the surgeon are: first, judgment; secondly, judgment; and thirdly, judgment. I wish to emphasize my point about laboratory diagnosis, and I therefore repeat: Never operate on a laboratory diagnosis unless the clinical evidence points in the same direction.

\section{ON Fractures.}

Next I wish to say a few words concerning fractures. To those of you who are in practice I suggest very strongly that you should never exclude a fracture or a dislocation until you have had the patient $\mathrm{X}$-rayed. Patients nowadays expect it, and if they do not expect it, the judge and jury do, if, later on, an action is brought against you for bad treatment. If your patient does not consent to have an X-ray taken, go straight home and write a letter to him strongly urging it, and keep a copy of the letter, otherwise you may be sued for not having had a skiagram taken. Do not be alarmed at the degree of deformity shown by X-rays, unless it corresponds with the degree of deformity which you detect clinically. There is no doubt that X-rays often exaggerate considerably the real extent of the deformity.

Another very important point is : Always control your treatment of. a fracture by radiography. It is not enough to have an $\mathrm{X}$-ray picture taken for diaspostic purpowen you must have one taken afterwards, nots much to satisfy yourself, as to guard against any action which may be taken against you afterwards if the result be not good. If you find that the bones are not in good position, 
try again. Sometimes you may have to make three or four attempts to put up a fracture before you get it into good position. When putting up a fracture I advise you always to give an anæsthetic; otherwise it is very difficult to get the fracture into good position.

Make a point of keeping the splints on the fracture until the union is firm. And remember that the periods of union are at least twice as long as those usually given in the textbooks. We learned that during the war. We are told in our textbooks that the period of union is three weeks for a humerus, and so on, but you should allow quite six weeks. Be sure that union is firm enough for the limb to take the weight of the patient. During the war it was not uncommon to send a patient away to a convalescent home with a splint on, then the surgeon or doctor in charge of the case said : "What is the good of a splint now ? It is so many weeks since the fracture, take it off." The result was that many patients came back with the limb bent at an acute angle because the soft callus which united the fracture gave way under the stress of walking and bearing weight. The great argument which is advanced in favour of taking the splints off is, that otherwise there will be a stiff joint. I say, don't worry about stiff joints; a stiff joint is better than an ununited fracture. I think the fear of stiff joints in the treatment of fractures is a bogie. I have never seen a permanently stiff joint simply from keeping the limb immobilized in the treatment of fracture. If a joint does acquire some stiffness because it is kept at rest, it soon recovers itself when the patient gets about on it, and when there has been a little passive movement.

As a general rule, oblique or spiral fractures have to be plated, especially in children. It is almost impossible to get a spiral or very oblique fracture into position by ordinary manipulation, and even if you do, it is almost impossible to keep it so. If you have ever seen an operation for plating a spiral fracture you will have noted that often there is extreme difficulty in getting the bones into position; and then when you have got them into position it is even more difficult to keep them so, long enough to apply a plate and screws. Much more, therefore, is it impossible to get and keep them into position when you are doing it by manipulation without being able to see what you are doing under the skin.

One piece of advice I want specially to impress upon you-I wish I could put it in such a way that you would never forget itis: Never plate a compound fracture; the attempt nearly always ends in disaster. Whatever the position is, however bad it is, let the wound heal up and see what happens, see what the result is after you have got healing. If necessary, you can do something at a later stage. If you plate a compound fracture it is almost certain to result in necrosis of a considerable part of the bone, and in some cases there is severe sepsis. I have known patients, as a result, not only lose their limb, but in one or two instances they have lost their life.

And lastly, do not make any rash promises as to the ultimate result. My old teacher, the late Mr. C. B. Lockwood, used to say : "It were better for a patient with a fracture to die than to have him walking about for the rest of his life with a badly set fracture."

\section{EMERgency Operations.}

Next, as regards emergency operations. Do not be in a hurry to operate in emergency cases without some preparation of the patient. There is rather a tendency, as soon as a diagnosis is made of appendicitis, or other acute condition, to operate on the patient, within half an hour, if possible. I am sure that is a great mistake; it is far better to spend some time in preparing the patient, giving sodium bicarbonate by the mouth, which makes him take the anæsthetic better, and administering continuous injection of saline and glucose by the rectum. I know only two operations in 
which delay must be cut down to the minimum; they are tracheotomy and perforation of a hollow viscus. In all other conditions I think it is wiser to delay operation from four to six hours, and in the few hours available to carry out as thorough treatment of the patient as you can. Further, 1 think that if you are wise you will not allow yourself to be persuaded, on the grounds of urgency, to operate in a private house. If the patient is fit for operation, he is fit to be moved to a hospital or a nursing home. The delay will be nıore than compensated by the greater facilities available, and hence the shorter time occupied in performing the operation. In cases of badly smashed limbs from railway or motor accidents, it is common for operation to be done immediately. I think that is wrong; one should wait until the patient has recovered from the initial shock, and these patients are more likely to recover from it if you leave them six, eight, or twelve hours. If you amputate a limb immediately on admission, when the patient is in a state of profound shock, the result is not likely to be so good. The reason given for immediate operation is that the limb may become septic, but as a rule a limb does not become septic in less than twenty-four hours, and that allows plenty of time to treat the patient for shock, and you can remove the limb before there is any serious degree of sepsis present.

\section{ACute Appendicitis.}

Next, with regard to acute appendicitis. Nowadays most people recognize that acute appendicitis requires immediate operation. You know Murphy's dictum : “ Now is the accepted time," and that is very true of acute appendicitis. There are a few surgeons and physicians who advise what they call, judging each case on its merits. I have never been able to discover quite what this means. If you ask them what they mean by it, you never get any very satisfac. tory answer. If you aver that you can jucige a case on its merits, it means not only that you can see what is going on inside the abdornen-which I cannot-but that you know what will happen there within the next two or three days-which I maintain nobody can tell. Until such time as we can envisage the course of the attack and what will happen in the near future, the only thing to do is to operate as soon as we reasonably can after the diagnosis has been made. But, here again, I am certain that cases of acute appendicitis do better if the operation is delayed from four to six hours, and, in the meantime, give plenty of saline by the rectum and sodium bicarbonate by the mouth, and prepare the skin thoroughly. I do not mind waiting, in some cases, eight or ten hours, to make sure that the patient has in him plenty of saline and glucose. At the British Medical Association meeting-I think it was in I9 I - there was a discussion on acute appendicitis. One surgeon maintained you could treat every case of appendicitis without operation, that they, sooner or later, all got well. In the discussion I asked him to give us the signs and symptoms by which he could judge each case on its merits, how he could tell what was going to happen in the next two or three days. There was, of course, no answer. There could not be an answer, because the age of prophecy is dead. We can only guess what is likely to happen. As to the operation, Murphy's dictum was, "get in quick, and get out quicker." Do not spend time over such things as invaginating the stump of the appendix. For years I invaginated the stump and changed my gloves before sewing up the abdominal wall; but little things like that are unnecessary because the case is already septic, and the changing of one's gloves before sewing up the skin cannot make a difference to the patient, and the stump of the appendix will heal up as well if you do not invaginate. It is an acute condition, and you want to get the operation done as quickly as possible; and invagina- 
tion occupies valuable time. A student was once asked to enumerate the structures encountered in exposing the appendix, and he replied : "Cut out all the cackle and come straight down on the appendix !" There was much wisdom in this reply.

Above all, in operating for acute appendicitis do not forget the drainage tube; it does no harm, and may save further operation. In some cases it saves the life of the patient. I am aware this is not in accordance with modern teaching, because the modern surgeon eschews the drainage tube; he says that not only are they unnecessary, that they do harm, that they leave the patient with a weak spot in the abdomenand there are other objections made to the tube. I would rather have a weak spot in my abdomen than lie in a wooden coffin, however comfortably. This teaching arises from the surgery of the war. A certain school of surgecns tried to dispute the immutable principle that sepsis requires drainage. If there is sepsis you must drain it. Beware of false gods. "When in doubt drain a wound" is a sound surgical motto, even in these enlightened days. And remember that sometimes drainage tubes are left in too long, though they are far more often taken out too soon. I said drainage tubes do no harm. In an experience of nearly thirty years I have only once seen a drainage tube do harm, and that was last year, in a case of perforation of duodenal ulcer. I put a drainage tube down in the pelvis, and two or three days later the patient began to vomit. He evidently had obstruction, and I opened him up, and found that a portion of intestine had insinuated itself inside the drainage tube, and had become gangrenous, and there was a local abscess. I removed the tube, excised a small piece of gangrenous gut, and he made a good recovery, and had no further trouble. I do not think one isolated case like that among many hundreds will shake my faith in the use of drainage tubes in cases of sepsis.

\section{Rectal Feeding.}

Rectal feeding is a scientific name for starvation. In every hospital pharmacopæia and in many textbooks, you will find magnificent formulæ for so-called nutrient enemata. I hope none of you will be deluded into imagining that these concoctions are ever absorbed. You have only to see the mess which is washed out of the rectum every day, because when a patient is given nutriment enemata, it is an article of faith that at the same time he must have the rectum washed out daily. If nutrient enemata are absorbed, why is it necessary to wash them out? One smell of the mess which is washed out will convince you that they are useless. Some years ago I analysed the contents of these washouts, and compared the analyses with those of the enemata which were administered, and I found that in the washouts there was much more nitrogen than had been put in in the enemata. So the only result was to make the patient excrete nitrogen into the horrible mess which was put into the rectum. I do not know how you can expect anything else, because the pelvic colon was never meant to digest or absorb a Lord Mayor's banquet. Plain saline, to which has been added half an ounce of glucose to the pint, will do all that is necessary, and it acts as a powerful stimulant.

\section{STRICTURE OF THE CESOPhagUS.}

Next, a few words about carcinoma of the œesophagus, causing stricture. Do not operate on every case of œosphageal stricture due to carcinoma. Remember that more patients die from bronchial complications than from starvation. The commonest cause of death in patients with carcinoma of the œesophagus is broncho-pneumonia. But when the patient has real difficulty in swallowing and cannot take enough nourishment by the mouth, I am sure his comfort is added to considerably by a properly performed jejunostomy. I say that because it 
is an operation which is far preferable to gastrostomy in the treatment of carcinoma of the œsophagus.

\section{INTERNAL HÆMORRHAGE.}

Do not decide in a hurry that operation is indicated in internal hæmorrhage. Nature can deal with it very efficiently if you will let her do so, and if you trust her she will rarely fail you. So far as hæmorrhage from gastric and duodenal ulcers is concerned, I do not believe it is ever wise to operate during the hæmorrhage. Beware of the fallacious argument which is sometimes put forward in regard to these cases, somewhat to this effect: This patient is bleeding, he will probably die, something must be done. But, even if we admit the premises are true and the patient will probably die, is there any reason whatever in doing something which will make him die sooner? I see no logic in that; and you cannot tell for certain that the patient will die, and in most of these cases of internal hæmorrhage operation may be the last straw for the patient who but for the operation might have recovered. There are many occasions in practice in which it is very difficult to decide against operation. To decide upon operation is much easier than to say in an emergency that you will not operate. To operate is easy, it is following the line of least resistance. To refuse to operate sometimes demands very considerable courage. To the true surgeon the satisfaction is infinitely greater when a patient is saved without the use of the knife than when an operation is done which the event proves to have been unnecessary. In many of these cases, if operation is performed for gastric or duodenal hæmorrhage, no cause for the hæmorrhage is found. There is nothing to be done but to close the abdomen. The operaton has been purely an exploratory one, and that is not very satisfactory, because you are merely adding a complication which may be just sufficient to prevent the recovery of the patient. Until last year $I$ had never seen a patient die from hæmorrhage from a gastric or duodenal ulcer ; it is a very rare event. In the one instance I have encountered death did not occur after the first hæmorrhage; this patient had had several hæmorrhages before she was admitted to the hospital; she had had so many that she had never quite rallied from the last, and she died.

\section{Acute Intestinal OBstruction.}

I think there is nothing more difficult to diagnose than acute intestinal obstruction in its initial stages; nothing easier in its later stages. Often the general practitioner is blamed most unjustly for not diagnosing it early. When I was house surgeon I used to think, "Why did not Dr. So and So diagnose this earlier ?" But during my early days I saw intestinal obstruction occur suddenly in a patient in hospital for another complaint, and since then I have never blamed the general practitioner, because it took me twenty-four hours to decide that there was obstruction, and by the time I had diagnosed it, it was too late. When a patient is seized suddenly with acute abdominal pain and vomiting, you should always suspect intestinal obstruction. And, above all, remember that a patient who can pass neither flatus nor fæces is a dying person.

Next, as regards operation in acute intestinal obstruction. The position here is different from that in internal hæmorrhage, because in the former death is inevitable without operation, therefore surgical intervention is justifiable even in the most desperate cases. You are justified in taking a big risk, even if it sometimes consists merely in opening the abdomen and draining the bowel, because there is no other alternative to save the patient.

\section{Ureteral Calculus.}

With regard to ureteral calculus, do not regard the $\mathrm{X}$-ray diagnosis of this as gospel ; 
calculi are not always there. Sometimes a calcified gland casts a shadow which is indistinguishable from that of a calculus. Even when there is a calculus in the ureter do not be in a hurry to operate, because it is probable that sooner or later the calculus will be passed. If you do decide to operate, be sure that the calculus is there at the time of your operation, because very often the mere shock of the patient thinking of the operation is enough to make him pass the stone. You should have a skiagram taken as near to the actual time of the operation as possible, and be sure that all the urine he passes is drained through muslin, to be sure that he does not pass a stone in the meantime. I have known a stone passed between the taking of a skiagram at I I.3O and 2 o'clock, the time fixed for the operation.

And, lastly, one word on saying, "I do not know." There are often occasions in one's life when one cannot make a diagnosis, when one is utterly baffled and does not know what the patient is suffering from. In those cases, I think, the best course is openly to say so. There is nothing to be ashamed of in not being able to tell what is going on inside the patient, and it is better to be open about it and say right out "I do not know." It is related that on one occasion the great Abernethy saw the son of a peer, whose case completely baffled him. In his blunt way he said to the father, "I don't in the least know what is the matter." "Well, Dr. Abernethy," said the peer, "if you don't know, is there anybody who can tell me?", "Yes, sir," replied Abernethy, "plenty, if you go to somebody who is sufficiently ignorant!"

\section{THE USES AND LIMITATIONS OF LIGHT TREATMENT.}

BEING A LECTURE DELIVERED

\author{
$B y$ F. D. HOWITT, \\ M.D., M.R.C.P.
}

AT THE PRINCE OF WALES GENERAL HOSPITAL.

(In connection with the Post-Graduate Course of The Fellowship of Medicine.)

THE first essential to be grasped about light treatment is the fact that it is merely an attempt to supply artificially a natural want. When a physician orders colchicum for gout, or salvarsan for syphilis, he is prescribing a drug which, either as the result of prolonged clinical experience, or of diligent research in the laboratory, has proved itself a valuable specific remedy for the particular disease in which it is employed. But the case of "light treatment" is different. It is not a specific remedy for a particular disease ; still less is it a specific remedy for every disease, as some of its protagonists would have us believe. It is merely a treatment designed to supply a natural want of the living beings.

If an animal is deprived of sunlight, either in quantity or degree, some pathological condition will follow just as surely as if it is deprived of food or water, air or exercise.

These facts were not unknown to the ancients, who appreciated very thoroughly the healing power of the sun, just as they realized it to be the source of life and energy. At Epidauros (the Temple of Esculapius) the outlines of a vast sanatorium, where sun baths, hydrotherapy, massage and other treatments on purely rational lines were carried out, can still be seen. But, like so many rational therapeutic measures, sun treatment seems to have been relegated to the background by the various strange principles which characterized medicine in the Middle Ages, only to be revived in recent times with greater fervour. And so, 